\title{
Could multidetector computed tomography play a role in the management of patients readmitted with recurrent chest pain after surgical coronary revascularization?
}

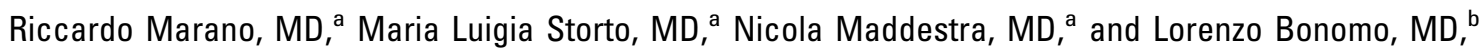 \\ Chieti and Rome, Italy
}

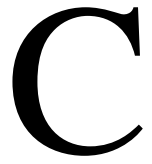
oronary artery bypass graft $(\mathrm{CABG})$ surgery is still one of the most performed surgical procedures worldwide, and arteriosclerotic graft disease is an important issue in cardiology, as shown by the increasing number of patients readmitted with recurrent chest pain after surgical revascularization or abnormal diagnostic test results. The availability of a noninvasive diagnostic tool to assess-exclude the presence of CABG disease could be of great clinical benefit for the management of patients. The imaging of coronary arteries by computed tomography has become reliable with the development of multidetector technology and the introduction of multidetector computed tomography (MDCT) at the end of the 1990s.

\section{Clinical Summary}

A 49-year-old man who had undergone CABG surgery 3 years previously was readmitted to the hospital for atypical angina and stress dyspnea. Table 1 illustrates the patient's baseline clinical parameters and graft typology. Echocardiography showed normal left ventricular wall kinesis; at rest, electrocardiographic (ECG) results were normal, whereas a stress test showed a slight $\mathrm{T}$-wave inversion in V4-6 without chest pain. For these reasons, the patient underwent retrospectively ECG-gated MDCT with the purpose of assessing the graft patency.

MDCT: Scanning. The examination was performed with a 4-row MDCT scanner (Somatom VZ; Siemens, Forchheim, Germany). With the patient in a supine position, peripheral venous access was prepared with a 20-gauge cannula for contrast material administration at the level of the right antecubital vein. After attachment of the leads for ECG recording, a noncontrast coronal localizer scan was performed to define the volume of the CT scan. Hence, MDCT angiography with retrospective ECG gating (HeartView, Siemens) was performed $(4 \times 2.5$-mm detector collimation,

From the Department of Clinical Science and Bioimaging, ${ }^{\text {a }}$ Section of Radiology, University "G. d'Annunzio," Chieti, Italy, and the Department of Bioimaging and Radiological Science, ${ }^{\mathrm{b}}$ Institute of Radiology, Catholic University, Rome, Italy

Received for publication Sept 23, 2004; accepted for publication Oct 12, 2004.

Address for reprints: Riccardo Marano, MD, Department of Clinical Science and Bioimaging, Section of Radiology, University "G. d'Annunzio" Chieti, Italy, Via dei Vestini 66100, Chieti, Italy (E-mail: r.marano@rad.unich.it).

J Thorac Cardiovasc Surg 2005;129:686-7

$0022-5223 / \$ 30.00$

Copyright $\odot 2005$ by The American Association for Thoracic Surgery doi:10.1016/j.jtcvs.2004.10.014
TABLE 1. Baseline characteristics of the patients and CABG typology assessed by means of retrospectively ECGgated MDCT

\begin{tabular}{lc}
\hline Age (y) & Male \\
Sex & No \\
Diabetes & No \\
Smoking history & No \\
Previous MI & No \\
Unstable angina & 63 \\
Heart rate (beats/min) & 125 \\
Systolic arterial pressure (mm Hg) & 75 \\
Diastolic arterial pressure (mm Hg) & 220 \\
Cholesterol (mg/dL) & 3 \\
Time since surgery (y) & (1) SVG to RCA; (2) LITA \\
CABGs & in situ to LAD; (3) \\
& RITA Y-grafted from \\
& LITA to OM
\end{tabular}

$M I$, Myocardial infarction; $C A B G$, coronary artery bypass graft; $S V G$, saphenous vein graft; $R C A$, right coronary artery; $L I T A$, left internal thoracic artery; $L A D$, left anterior descending artery; RITA, right internal thoracic artery; $O M$, obtuse marginal branch.

3-mm slice width, and $1.5-\mathrm{mm}$ reconstruction increment) to scan from the origin of the left internal thoracic artery (LITA) to the cardiac base within a single breath hold. One hundred ten milliliters of nonionic contrast material (Iomeron $300 \mathrm{mgI} / \mathrm{mL}$; Bracco, Milan, Italy) was administered with an automatic injector (Medrad-EnVision CT, Pittsburgh, Pa) at a flow rate of $3 \mathrm{~mL} / \mathrm{s}$; the start delay of the scan was fixed at 25 seconds. The right arm was chosen as the site of injection to avoid artifacts hampering the LITA takeoff because of the high contrast material concentration in the left innominate vein. The patient's heart rate was 63 beats/ min with sinus rhythm, and it was not necessary to reduce it with oral or intravenous $\beta$-blockers.

MDCT: Image reconstruction. MDCT angiography was performed without complication, and raw data were reconstructed in the mid-to-late diastolic phase to obtain free-motion images for further postprocessing. Hence CT axial images were transferred to an online workstation (Leonardo, Siemens) to perform bidimensional multiplanar reconstruction and maximum intensity projection images to obtain a complete visualization of the grafts, the recipient vessels, or both.

\section{Results}

MDCT showed regular patency of the saphenous vein graft to the right coronary artery with retrograde filling and distal patency of 


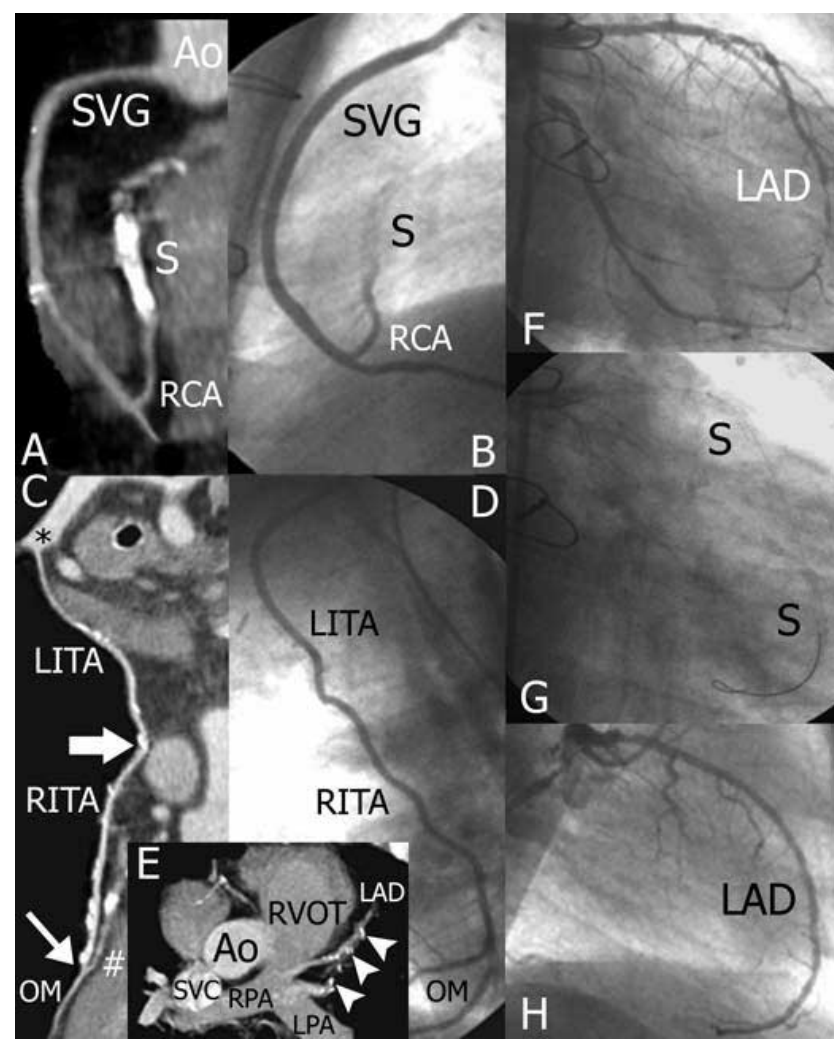

Figure 1. Curved multiplanar reconstruction of the saphenous vein graft to the right coronary artery (A), with good visualization of the distal anastomosis and retrograde filling of the native coronary vessel, as demonstrated by the corresponding coronary angiography (B). Curved multiplanar reconstruction (C) of a whole patent Y-graft right internal thoracic artery visualized from the proximal anastomosis on the left internal thoracic artery (thick arrow) to the distal anastomosis on the obtuse marginal (thin arrow). Lack of enhancement (arrowheads) of the left internal thoracic artery along the surgical clips (E) distally the right internal thoracic artery takeoff caused by total occlusion, as shown by the coronary angiography (D). Coronary angiography showed multiple stenotic lesions of the middistal left anterior descending artery (F). The post-percutaneous coronary angioplasty and stenting ( $G$ and $H$ ) angiographic control confirmed a good result of the procedure with patency of the coronary vessel. Ao, Aorta; $L A D$, left anterior descending artery; LITA, left internal thoracic artery; $L P A$, left pulmonary artery; $O M$, obtuse marginal branch; $R C A$, right coronary artery; $R I T A$, right internal thoracic artery; $R P A$, right pulmonary artery; $R V O T$, right ventricular outflow tract; $S$, coronary stent; $S V C$, superior vena cava; $S V G$, saphenous vein graft. *Left subclavian artery; \#left circumflex artery. the coronary artery (Figure $1, A$ ), as well as normal patency of the Y-graft right internal thoracic artery (RITA) from the LITA to the obtuse marginal branch (Figure 1,C). The LITA was well visualized from its origin to the proximal RITA graft's takeoff (Figure 1, $C$ ), whereas the lack of vascular enhancement resulted in its distal portion along surgical clips because of complete occlusion of the graft (Figure 1,E). For these reasons, the patient underwent coronary angiography, which confirmed MDCT findings (Figure 1, $B$ and $D$ ), showing severe lesions in the middistal left anterior descending artery (Figure $1, F$ ), and therefore percutaneous angioplasty and stenting of the left anterior descending artery were performed (Figure 1, $G$ and $H$ ).

\section{Discussion}

Survival late after CABG surgery is largely dependent on graft patency and progression of coronary artery disease. The grafts are vulnerable to arteriosclerosis, with a lower long-term patency of venous than arterial grafts. Invasive coronary angiography is still the standard of reference for the assessment of the status of CABGs and recipient vessels; however, it requires hospitalization and arterial catheterization, with a small risk of complications. In the last years, diagnostic techniques have been tested for noninvasive imaging of CABGs, as well as coronary arteries. ${ }^{1-3}$ Limited scan coverage, clinical availability, prospective ECG triggering, and higher cost have limited electron beam computed tomography. In the 1990s, the development of the MDCT scanner and the availability of software for retrospective ECG gating partly overcame these limitations, and it has recently shown high accuracy in the detection of coronary artery disease. ${ }^{4}$ There are still few reports in the recent literature ${ }^{5}$ showing a potential role of MDCT in the assessment of patients undergoing surgical coronary revascularization. In our opinion this case could strengthen the role of this noninvasive diagnostic tool in selected patients with unconvincing stress test results, with a great benefit for the clinical management of these patients.

\section{References}

1. Ha JW, Cho SY, Shim WH, Chung N, Yang Y, Lee HM. Non invasive evaluation of coronary artery bypass graft patency using three dimensional angiography obtained with contrast enhanced electron beam CT. Am J Roentgenol. 1999;172:1055-9.

2. Wintersperger BJ, Engelmann MG, von Smekal A, Knez A, Penzkofer HV, Hofling B. Patency of coronary by-pass grafts: assessment with breath-hold contrast enhanced MR angiography-value of a non electrocardiographically triggered technique. Radiology. 1998;208:345-51.

3. Langerak SE, Vliegen HW, de Roos A, Zwinderman AH, Jukema JW, Knuz P. Detection of vein graft disease using high-resolution magnetic resonance angiography. Circulation. 2002;105:328-33.

4. Ropers D, Baum U, Pohle K, Anders K, Ulzheimer S, Ohnesorge B. Detection of coronary artery stenoses with thin-slice multi-detector row spiral computed tomography and multiplanar reconstruction. Circulation. 2003;107:664-6.

5. Marano R, Storto ML, Maddestra N, Bonomo L. Non invasive assessment of coronary artery bypass graft with retrospectively ECG-gated four-row multi-detector spiral computed tomography. Eur Radiol. 2004;14:1353-62. 